\title{
HIGH ANGULAR RESOLUTION OBSERVATIONS OF
}

\section{PROTOSTELLAR DISKS}

\author{
J.-L. MONIN, J. BOUVIER and F. MALBET \\ Observatoire de Grenoble, Laboratoire d'Astrophysique, 414 rue de la piscine, BP $53 X$, \\ 38041 Grenoble Cedex, France
}

january 1993

\begin{abstract}
.
The existence of circumstellar disks around young stellar objects like $T$ Tauri stars is now well accepted. Such disks would have solar system sizes and, at the distance of the nearest star forming cloud, an angular diameter of 0.01 to 1 arcsecond at most, requiring very high angular resolution to be detected. Due to the nature of the emission process in circumstellar disks and to chromatic properties of ground based observations, disk imaging is expected to be more efficient in the near infrared. Also, multi-aperture interferometry in this wavelength range $(1-10 \mu \mathrm{m})$ is expected to bring considerable insight into the disks properties and evolution in revealing their inner physical structure.

In this paper, we present synthetic images of circumstellar accretion disks. The images have been computed from a complete disk vertical structure model.
\end{abstract}

Key words: Young stars, circumstellar disks, accretion, thermal emission, scattering, imagery, interferometry.

\section{INTRODUCTION}

Recently, direct evidence for circumstellar disks was gained from high angular resolution imaging, using either speckle interferometry or adaptive optics. The detected light originates from the scattering of stellar photons in the circumstellar material (Monin et al., 1989; Malbet et al., 1993). At the distance of the nearest star forming cloud $(\approx 100 \mathrm{pc})$, the inferred angular size of circumstellar disks falls in the subarcsecond range. Moreover, emission in the optical range (UV to mid-IR) arises from the inner parts of the disk and requires a spatial resolution that can only be attained by large telescopes and / or by multi-aperture interferometry.

Bertout \& Bouvier (1988) have computed synthetic images of protoplanetary disks in the infrared for the Very Large Telescope Interferometer by taking into account only thermal emission and assuming infinitively flat disks. Lazareff et al. (1990) considered scattered light in different analytical models. Ménard $(1989,1992)$ derived polarisation maps resulting from multiple scattering in circumstellar dust around young stars. Whitney \& Hartmann $(1992,1993)$ also performed polarisation and intensity maps but with a lower spatial resolution. Finally, Malbet et al. (1992) gave a preliminary report of this work with the difference that reprocessing was not yet included in the computations.

In this paper, we present synthetic images computed from a disk model based on the classical accretion disk model, with the additional computation of the vertical density and temperature structure of the disk. We consider : (I) thermal emission from the accretion disk, (II) thermal emission due to the heating by the central star, (III) single scattering in the upper, optically thin parts of the disk atmosphere.

Images at various lines of sight and wavelengths (from visible to far infrared) are 
computed. These synthetic maps can be used as a guide to interpret the results of current high angular resolution imaging of the immediate environment of young stars. A complete series of such images will be presented in a forthcoming paper.

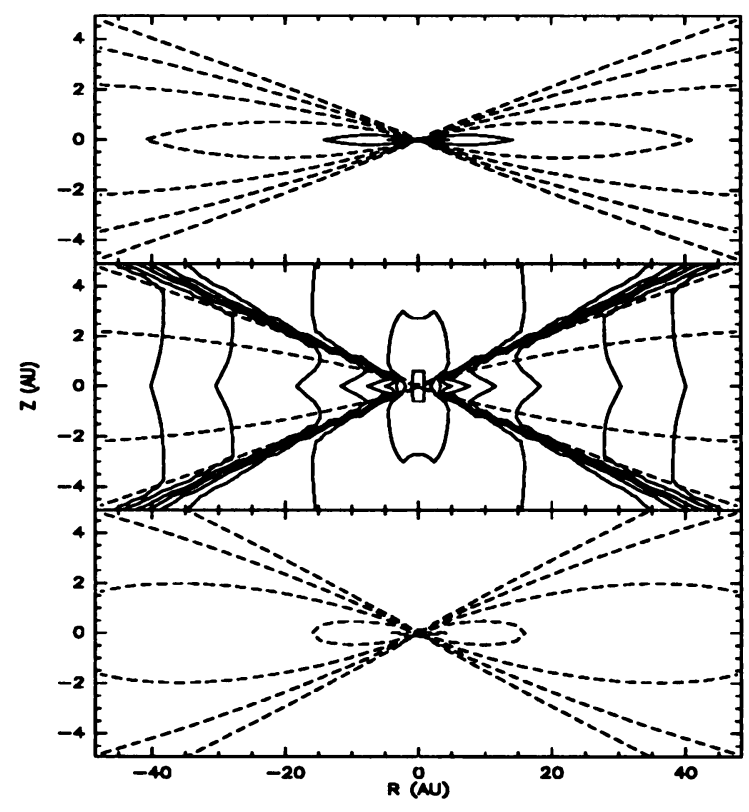

Fig. 1. Vertical structure of a circumstellar disk around a young low-mass star. Upper panel: optical depth contours in logarithmic scale (from $\tau=10^{-4}$ to $\tau=10$ by step of 10) showing optically thick regions (solid line) and optically thin regions (dashed line) of the disk. Middle panel: temperature contours (solid line, levels $: \log T=1.2,1.4,1.6,1.8,2.0$, 2.25, 2.5 and 2.75), and optical depth contours for $\tau=10^{-2}$ and $\tau=10^{-4}$ (dashed line). Lower panel: logarithmic density contours (from $\rho=10^{-15} \mathrm{~g} \cdot \mathrm{cm}^{-3}$ to $\rho=10^{-10} \mathrm{~g} \cdot \mathrm{cm}^{-3}$ by steps of 10).

\section{DISK MODEL}

We first use the classical accretion disk model of Lynden-Bell \& Pringle (1974) to compute temperature and density radial distributions, averaged in the vertical direction. We then solve the radiative transfer in the $z$-direction as in a planeparallel atmosphere at each radius. We use the set of parameters described in table I, with a $1 \mathbf{M}_{\odot}$ star. We compute the vertical structure using the opacities given by Lin \& Papaloizou (1985), and use the monochromatic opacities given by Wolfire \& Cassinelli (1986) for a mixture of graphite and silicates, to compute the emission of the grains in various photometric bands of the near to mid infrared. For the disk's viscosity, we use the $\alpha$ prescription (Shakura \& Sunyaev, 1973) 


\begin{tabular}{|l||l|l|}
\hline Stellar parameters & $R_{*}$ & $2 \mathrm{R}_{\odot}$ \\
& $T_{*}$ & $4500 \mathrm{~K}$ \\
\hline \hline Disk parameters & $R_{\min }$ & $1.1 R_{*}$ \\
& $R_{\max }$ & $200 A U$ \\
& $\dot{M}_{\text {acc }}$ & $10^{-7} \mathrm{M}_{\odot} \mathrm{yr}^{-1}$ \\
& $\alpha_{\text {visc }}$ & 0.1 \\
\hline
\end{tabular}

TABLE I

Model parameters

The emergent intensity on a line of sight $\left(x_{\text {obs }}, y_{\text {obs }}\right)$ on the plane of the sky is computed as:

$$
I_{\nu}\left(x_{\text {obs }}, y_{\text {obs }}\right)=\int_{0}^{\infty}\left[(1-\varpi) B_{\nu}(T(r, z))+\frac{\varpi}{4 \pi} \frac{\pi R_{*}^{2}}{d^{2}} B_{\nu}\left(T_{*}\right) e^{-\tau_{*}(r, z)}\right] e^{-\tau_{\text {obs }}} \mathrm{d} \tau_{\text {obs }}
$$

We have computed intensity maps from 0.5 to $500 \mu \mathrm{m}$ for each of the three emission processes considered in the model: viscous heating, reprocessing, and scattering. At $2.2 \mu \mathrm{m}$, scattering dominates on the large scale while thermal emission due to either viscous heating or reprocessing is limited to the innermost disk regions. At $20 \mu \mathrm{m}$, thermal emission due to viscous heating is still limited to the inner 10 $\mathrm{AU}$ of the disk, though more extended than at $2.2 \mu \mathrm{m}$, while reprocessing now contribute more than scattered light within $100 \mathrm{AU}$ from the star. At both 2.2 and $20 \mu \mathrm{m}$, thermal emission due to reprocessing extends further away from the star than thermal emission due to viscous heating as a result of disk flaring. A dark lane can be seen in the disk's equatorial plane, resulting from the enhanced opacity in the disk's densest layers.

Our model shows that scattering dominates at short wavelengths while thermal emission becomes important at long wavelengths. At $\lambda=10 \mu \mathrm{m}$, scattering is greatly reduced and thermal emission is not yet important, while at $\lambda=20 \mu \mathrm{m}$, thermal emission, and in particular reprocessing, becomes the major source of flux in the inner disk regions.

Figure 2 shows intensity maps at $\lambda=2.2 \mu \mathrm{m}$ are shown for an inclination of 0,60 , 75 , and $90^{\circ}$. At this wavelength, single scattering is the main source of light over the whole disk structure. It is seen from the edge-on image that scattering occurs only in the upper and lower optically thin edges of the disk while no scattering occurs in the optically thick, equatorial disk's plane. Our model shows that scattering is easier to detect at short wavelengths while thermal emission is more easily detected at mid- and far-infrared wavelengths because of the smoother variation of the radial brightness profiles.

The primary constraint for detecting thermal emission is spatial resolution while a high dynamical range is mandatory to detect scattered light. 


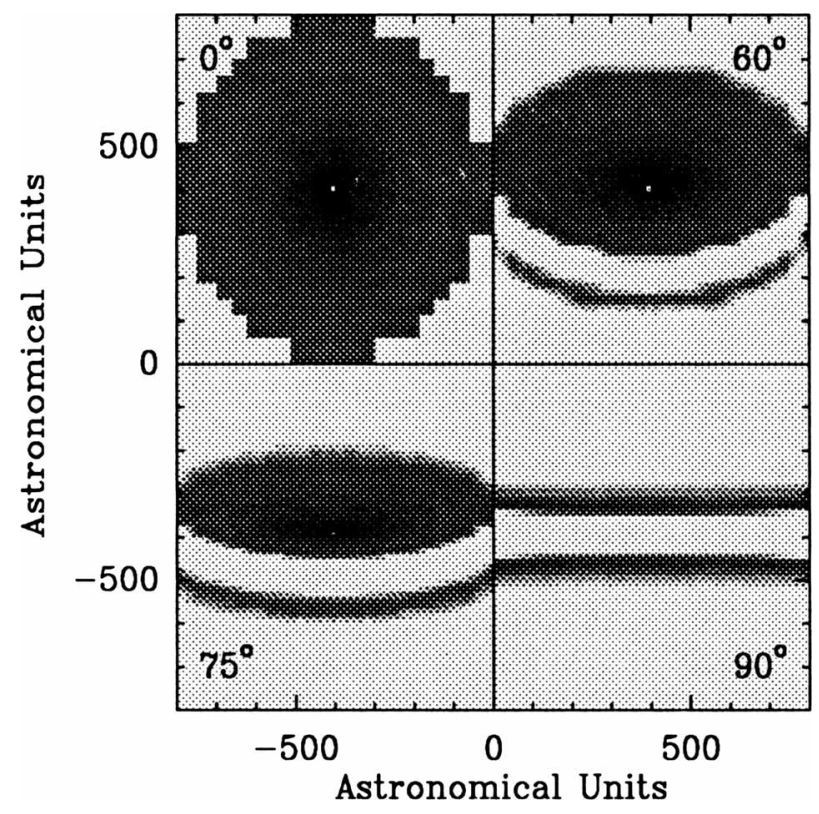

Fig. 2. Dependence upon inclination angle at $\lambda=2.2 \mu \mathrm{m}$.

\section{References}

Bertout, C., Bouvier, J. 1988, Interferometric Imaging of Protoplanetary Disks around Young Stars, Proc NOAO-ESO Conf on High-Resolution Imaging by Interferometry, ed. F. Merkle, 69.

Lazareff, B., Pudritz, R.E., Monin, J-L. 1990, Infrared Images of Protostellar Accretion Disks: Theoretical Models, Astrophys Journal, 358, 170.

Lin, D. N. C., Papaloizou, J. 1985, On the Dynamical Origin of the Solar System, in Protostars and Planets II, ed. Black, D. C., Matthews, M. S. (Tucson: University of Arizona Press), 493.

Lynden-Bell, D., Pringle, J. E. 1974, The Evolution of Viscous Discs and The Origin of the Nebular Variables, Monthly Notes of Royal Astron Soc, 168, 603.

Malbet F., Monin J.-L., Bouvier J. (1992), 'Imaging Circumstellar Disk around Young Stars by Multi-Aperture Interferometry', Proc. ESA Coll. Targerts for Space-Based Interferometry, Beaulieu, Oct. 92, in press

Malbet, F., Rigaut, F., Bertout, C., Léna, P. 1992, Detection of a 400 AU disk-like structure around the young stellar object $\mathrm{Z} \mathrm{CMa,} \mathrm{submitted} \mathrm{to} \mathrm{Astron} \mathrm{Astrophys.}$

Ménard, F., 1989, Etude de la polarisation causée par des grains dans les enveloppes circumstellaires denses, $P h D$ Thesis, Univ. of Montreal.

Ménard, F., 1992, Astrophys Journal, submitted.

Monin, J-L., Pudritz, R.E., Lacombe, F., Rouan, D. 1989, Infrared Images of HL Tau: Scattering from an Inclined, Flaring Disk, Astron Astrophys, 215, L1.

Shakura, N.I., and Sunyaev, R.A. 1973, Black Holes in Binary Systems. Observational Appearance, Astron Astrophys, 24, 337.

Whitney, B.A., Hartmann, L. 1992, Model scattering envelopes of Young Stellar Objects. I. Method and application to circumstellar disks, Astrophys Journal, in press

Whitney, B.A., Hartmann, L. 1993, Model scattering envelopes of Young Stellar Objects. I. Infalling envelopes, Astrophys Journal, submitted.

Wolfire, M.G., Cassinelli, J.P. 1986, The Temperature Structure in Accretion Flows onto massive Protostars, Astrophysical Journal, 310, 207 